УДК 517.9

\title{
Monte Carlo Simulation of Magnetic Multilayered Structures with the Effects of Giant Magnetoresistance
}

\author{
Dmitriy E. Romanovskiy* \\ Marina V. Mamonova ${ }^{\dagger}$ \\ Vladimir V. Prudnikov $\ddagger$ \\ Pavel V. Prudnikov ${ }^{\S}$ \\ Omsk State University \\ Mira, 55a, Omsk, 644077 \\ Russia
}

Received 20.08.2016, received in revised form 10.10.2016, accepted 14.11.2016

Monte Carlo simulations of magnetic properties for structures, which are constructed from two ferromagnetic films divided by nonmagnetic film, are carried out using the anisotropic Heisenberg model. The value for exchange integral for $\mathrm{Fe}(100) / C r$ system was calculated using PAW PBE approximation. The calculation of the magnetoresistance coefficient is carried out for different thicknesses of the ferromagnetic films in case of CPP geometry. It was shown, that the obtained temperature dependence for the magnetoresistance coefficient is agreed very well with experimental results, measured for the $\mathrm{Fe} / \mathrm{Cr}$ and Co/Cu multilayered structures.

Keywords: effect of giant magnetoresistance, magnetic multilayered structures, ultrathin films, magnetoresistance coefficient, anisotropic Heisenberg model, Monte Carlo methods.

DOI: 10.17516/1997-1397-2017-10-1-65-70.

\section{Introduction}

The ultrathin films of magnetic metals and alloys are used as a components for magnetic multilayer structures. These artificial created magnetic structures has become of great interest for wide range of applications based on the phenomena of the giant magnetoresistance (GMR).

The multilayer structures with GMR effects consist of the ferromagnetic layers separated by nonmagnetic layers. The thickness of these nonmagnetic metal layers are selected in such a way that the long-range and oscillating RKKY exchange interaction between the spins of the ferromagnetic layers is effective antiferromagnetic. Through this interaction, the magnetizations of the adjacent ferromagnetic layers are oriented opposite to each other. When this structure is placed in an external magnetic field, the magnetization of layers begin to orient in parallel, that leads to a significant change in electrical resistance. Devices based on the GMR effect are widely used as read heads of hard disks, memory devices and spintronic devices [1].

\footnotetext{
*romfizz898@gmail.com

†mamonova-mv@mail.ru

${ }^{\ddagger}$ prudnikp@univer.omsk.su

$\S$ prudnikp@univer.omsk.su

(c) Siberian Federal University. All rights reserved
} 


\section{Model}

For the description of magnetic properties of the ultrathin films $\mathrm{Fe}$ and Co at structures $\mathrm{Fe} / \mathrm{Cr}$ and $\mathrm{Co} / \mathrm{cu}$ we introduce the Hamiltonian of the spin system in the form:

$$
H=-J_{1} \sum_{i, j}\left\{\left[1-\triangle_{1}(N)\right]\left(S_{i}^{x} S_{j}^{x}+S_{i}^{y} S_{j}^{y}\right)+S_{i}^{z} S_{j}^{z}\right\}
$$

for the out of plane magnetisation $(\mathrm{Fe} / \mathrm{Cr}) \mathrm{e}$ and

$$
H=-J_{1} \sum_{i, j}\left\{\left(S_{i}^{x} S_{j}^{x}+S_{i}^{y} S_{j}^{y}\right)+\left[1-\triangle_{2}(N)\right]\left(S_{i}^{z} S_{j}^{z}\right)\right\},
$$

for the in plane magnetisation $(\mathrm{Co} / \mathrm{Cu})$. In $(1)$ and $(2)$ relations, $J_{1}>0$ characterizes the shortrange exchange interaction between the spins, $S_{i}=\left(S_{i}^{x}, S_{i}^{y}, S_{i}^{z}\right)$ is a three-dimensional unit vector, $\triangle_{1,2}(N)$ are an anisotropy parameters. The dependence of the anisotropy parameters $\triangle_{1,2}(N)$ on the film thickness $N$ is selected proportional to dependence of the critical temperatures for Fe and Co films on $N$ (Fig. 1a and b). The simulations were performed for ferromagnetic films with the body-centered (Fe) and face-centered $(\mathrm{Co})$ cubic structures characterized by linear sizes $L \times L \times N$ and periodic boundary conditions in the film plane (Fig. 1c).
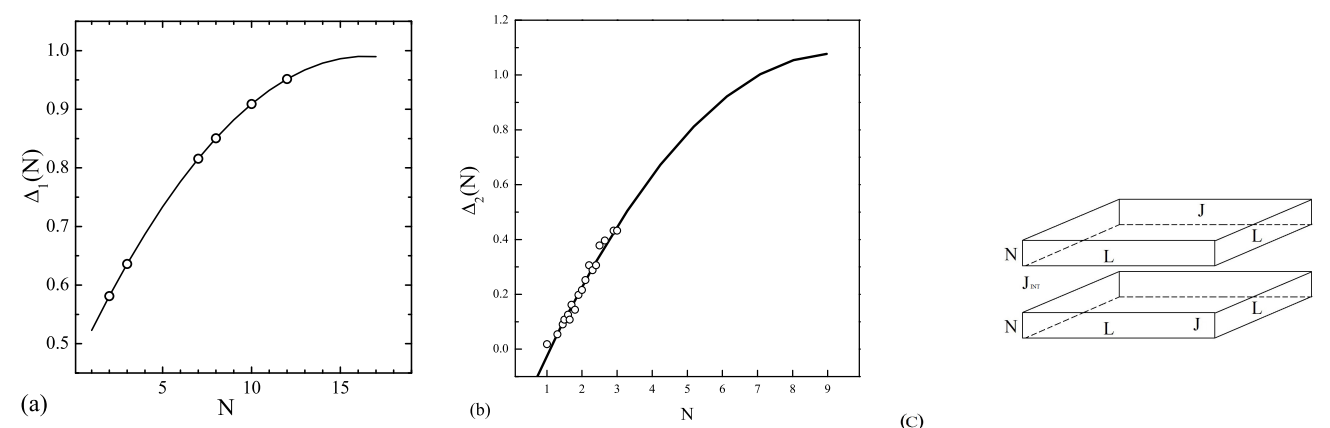

Fig. 1. Dependence of the anisotropy parameters $\triangle_{1,2}$ on the ferromagnetic film thickness $N$ for systems $\mathrm{Fe} / \mathrm{Cr}(100)$ (a) and $\mathrm{Co} / \mathrm{Cu}(100)$ (б). The circles are experimental data, which were used in calculations. The model of the multilayer structure (c) which consists of two ferromagnetic films separated by nonmagnetic metal film; $L$ and $N$ are linear sizes of films, $J_{1}, J_{\text {int }}$ are the exchange integrals

\section{The calculation of the exchange integral}

The value of the exchange integral that determine the interaction between neighboring atoms of $\mathrm{Fe}$ in the layered $\mathrm{Fe} / \mathrm{Cr}$ superlattices was calculated with the use of the VASP programm package. The calculations of the total energy were realized for different ferromagnetic and antiferromagnetic spin configurations within the bounds of PAW PBE approximation. The contribution to ab initio total energies, corresponding to exchange interaction, can be described by the following classical Heisenberg model:

$$
H=-\sum_{i, j} J_{i j}\left(\mathbf{S}_{i} \mathbf{S}_{j}\right)
$$


For the bcc Fe-based systems, the exchange interaction is relatively short ranged, so the main contribution to the exchange energy comes from the interaction up to next-nearest neighbours. The antiferromagnetic spin configuration for which the calculation was carried out is shown in Fig. 2. The magnetic moment of atoms is directed along the $z$ axis. We got a system of equations for determination of $J_{1}$ and $J_{2}$ by calculating the difference in energies of the ferromagnetic and antiferromagnetic configurations of spins in the film. The coefficients of linear equations are equals to the number of pairs of oppositely directed magnetic moments of the nearest and nextnearest atoms.
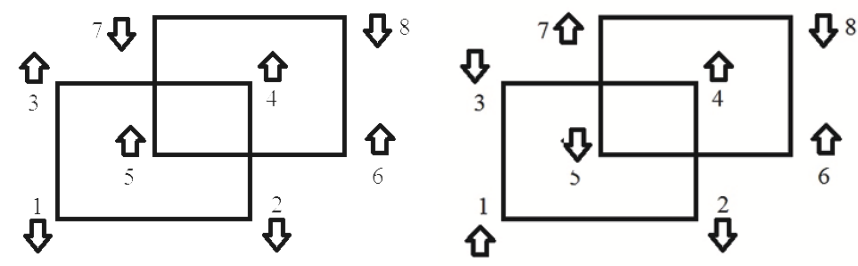

Fig. 2. Antiferromagnetic spin configuration for two adjacent films which are used for the calculation of the exchange integrals in $\mathrm{Fe} / \mathrm{Cr}$ structure

Bonds for the nearest atoms are formed for atoms with the numbers $1-3 ; 1-2 ; 3-4 ; 2-4 ; 5-6$; $5-7 ; 7-8 ; 8-6$. Bonds for the next-nearest atoms are formed for atoms with the numbers $1-5 ; 2-5$; $2-6 ; 3-5 ; 3-7 ; 4-5 ; 4-6 ; 4-7 ; 4-8$.

A system consisting of two ferromagnetic films of iron Fe(100) with 3 monolayer thickness, separated by non-magnetic film of $\mathrm{Cr}(100)$ with 3 monolayer thickness also:

$$
\left(\begin{array}{ll}
20 & 24 \\
24 & 12
\end{array}\right)\left(\begin{array}{l}
J 1 \\
J 2
\end{array}\right)=\left(\begin{array}{c}
3,9043 e V \\
3,766 e V
\end{array}\right) .
$$

As a result, we received values $J_{1}=1.73 \cdot 10^{-14} \mathrm{erg}, J_{2}=0.73 \cdot 10^{-14} \mathrm{erg}$.

The similar ab initio calculations were realized for system $\mathrm{Co} / \mathrm{Cu}(100) / \mathrm{Co}$ consisting of two ferromagnetic films of iron Fe(100) with 3 monolayer thickness, separated by non-magnetic film of $\mathrm{Cr}(100)$ with 3 monolayer thickness also. We determined the values of the exchange integral $J_{1}=2.52 \cdot 10^{-14} \mathrm{erg}$ for interaction of the nearest neighbours and $J_{2}=1.68 \cdot 10^{-14} \mathrm{erg}$ for interaction of the next-nearest neighbours.

\section{Simulation algorithm and results of calculations}

The method was developed for the calculation of the magnetoresistance coefficient for multilayered structures in case of CPP-geometry [2].

We denote the resistance of a ferromagnetic film for two groups of electrons with spins up and down as $R_{\uparrow}$ and $R_{\downarrow}$, respectively, and use the simple two-current Mott model for description of the resistance of different conductance channels for calculation of the magnetoresistance $\delta_{h}$ of the trilayer structure. Within the bounds of the two-current model we neglect the scattering of current carriers from interfaces and the resistance of the nonmagnetic metal in comparison with the resistance of ferromagnetic metal. The magnetoresistance of trilayer structure is determined by relation

$$
\delta_{h}=\frac{\left(R_{\uparrow}-R_{\downarrow}\right)^{2}}{4 R_{\uparrow} R_{\downarrow}}=\frac{\left(\mathbf{J}_{\uparrow}-\mathbf{J}_{\downarrow}\right)^{2}}{4 \mathbf{J}_{\uparrow} \mathbf{J}_{\downarrow}},
$$


where $\mathbf{J}_{\uparrow, \downarrow}=e n_{\uparrow, \downarrow}\left\langle\mathbf{V}_{\uparrow, \downarrow}\right\rangle$ is the current density. Hear, $n_{\uparrow, \downarrow}$ is the density of electrons with spin moment equal to $+1 / 2$ and $-1 / 2, n=n_{\uparrow}+n_{\downarrow}$ is the total electron density, $\left\langle\mathbf{V}_{\uparrow, \downarrow}\right\rangle$ are the averaged velocity of electrons with corresponding spin projections. The electron densities with spin up and down can be expressed through the magnetization of film $n_{\uparrow, \downarrow} / n=(1 \pm m) / 2$. The averaged electron velocity $\left\langle\mathbf{V}_{\uparrow, \downarrow}\right\rangle$ can be expressed through an electron mobility and the external electric field intensity $\mathbf{E}$, and after that through a probability of electron jump in unit time from $i$-cell to a neighbouring cell in the direction of electric field [2] with averaging over all film cells:

$$
\left\langle\mathbf{V}_{\uparrow, \downarrow}\right\rangle=\mu_{\uparrow, \downarrow} \mathbf{E}=\frac{e}{T} \mathbf{E}\left\langle\exp \left(-\frac{\Delta E_{i, \uparrow, \downarrow}}{T}\right)\right\rangle,
$$

where $\mu$ is the electron mobility, $\Delta E_{i}$ characterizes the change of system energy connected with electron jump from $i$-cell to a neighbouring cell. $E_{i, \uparrow, \downarrow}$ is determined by relation

$$
E_{i, \uparrow, \downarrow}=\mp J_{1}\left[\sum_{j \neq i} S_{j}^{z}\left(n_{j, \uparrow}-n_{j, \downarrow}\right)+S_{i}^{z}\left(n_{i, \uparrow}-n_{i, \downarrow}\right)\right],
$$

where the summation is realized of cells $j$ neighbouring to $i$ cell.

On the basis of the above presented relations, we calculated the temperature dependence of the magnetoresistance for the trilayer structures $\mathrm{Fe} / \mathrm{Cr}(100) / \mathrm{Fe}$ and $\mathrm{Co} / \mathrm{Cu}(100) / \mathrm{Co}$ with different thicknesses $N$ of ferromagnetic films. The graphs of calculated temperature dependence of the magnetoresistance are given in Fig. 3 and Fig. 4 for trilayer magnetic structures $\mathrm{Fe} / \mathrm{Cr}(100) / \mathrm{Fe}$ and $\mathrm{Co} / \mathrm{Cu}(100) / \mathrm{Co}$ for different thicknesses of ferromagnetic films $N$ with the anisotropy parameters $\triangle_{1,2}(N)$ given in Fig. 1a, b and the values of exchange integral $J_{1}=2 \cdot 10^{-14} \mathrm{erg}$ for Fe and $J_{1}=4.4 \cdot 10^{-14} \mathrm{erg}$ for Co obtained from mean-field estimations. The dependence of the magnetoresistance on the thickness $N$ for the $\mathrm{Fe} / \mathrm{Cr} / \mathrm{Fe}$ structure corresponds to dimensional changes of the critical temperature of ferromagnetic phase transition in Heisenberg films revealed in paper [4] and agree with results of the experimental investigations of dimensional phenomena in the ultrathin films Fe. The calculated temperature dependence of the magnetoresistance agrees very well with experimental data with CPP-geometry [3].
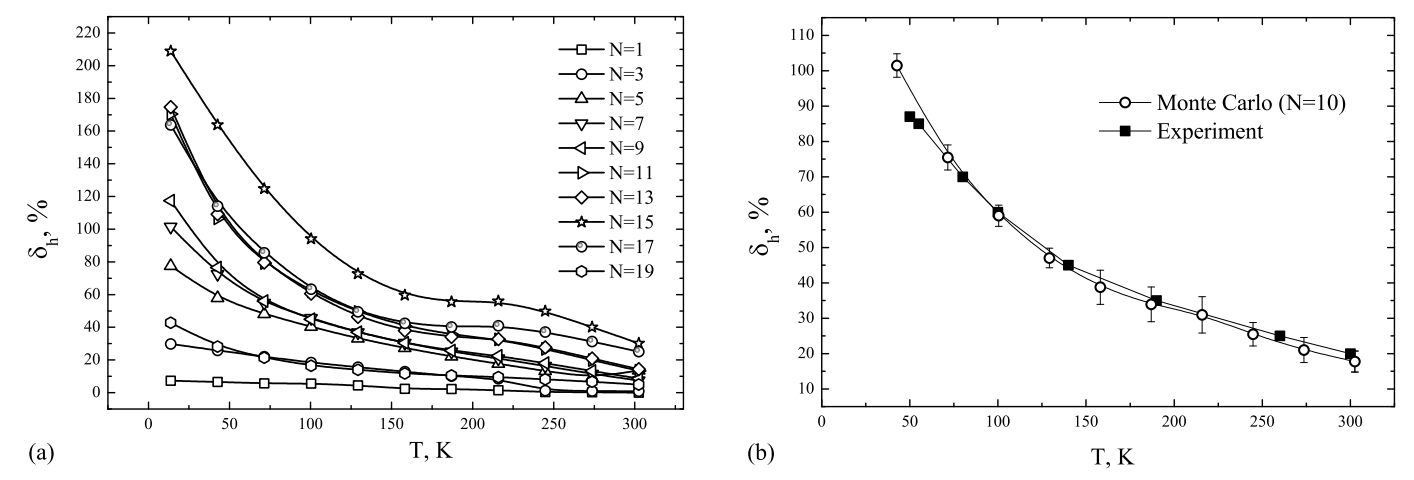

Fig. 3. The dependence of the magnetoresistance of the trilayer structure $\mathrm{Fe} / \mathrm{Cr}(100)$ on the temperature for different values of the thickness of Fe films (a); comparison of the calculated and experimentally measured in [3] the temperature dependence of the magnetoresistance with the thickness of the Fe films $4 \mathrm{~nm}(N=10)$ (b) 

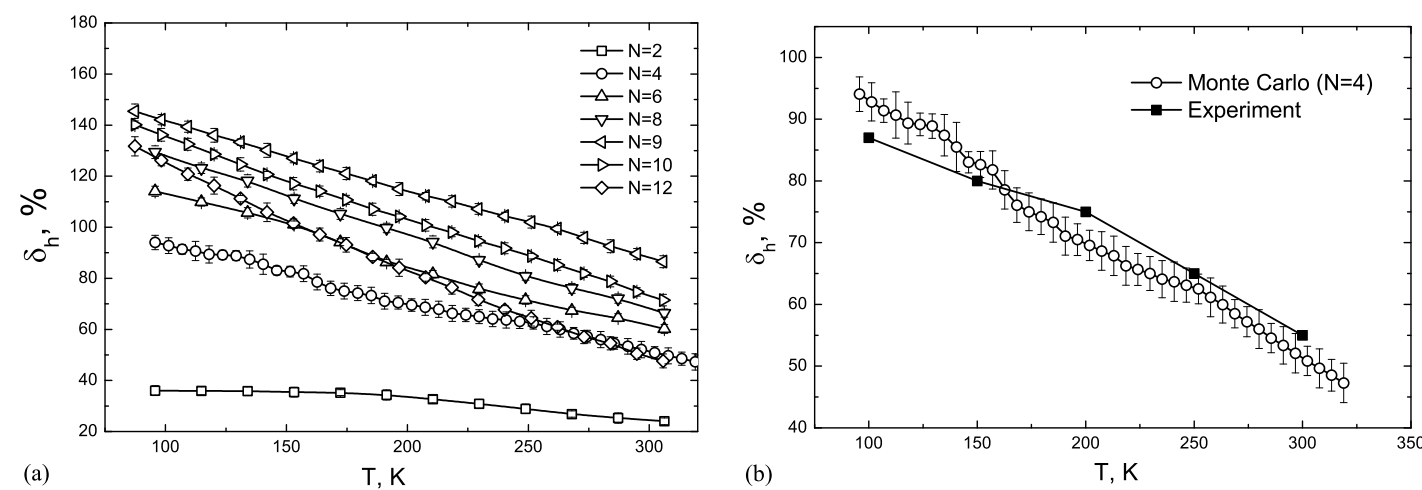

Fig. 4. The dependence of the magnetoresistance of a three-layer structure $\mathrm{Co} / \mathrm{Cu}(100)$ on the temperature for different values of the thickness of Co films (a), comparison of the calculated and experimentally measured in [3] temperature dependence of the magnetoresistance with the thickness of the Co films $1.2 \mathrm{~nm}(N=4)(\mathrm{b})$
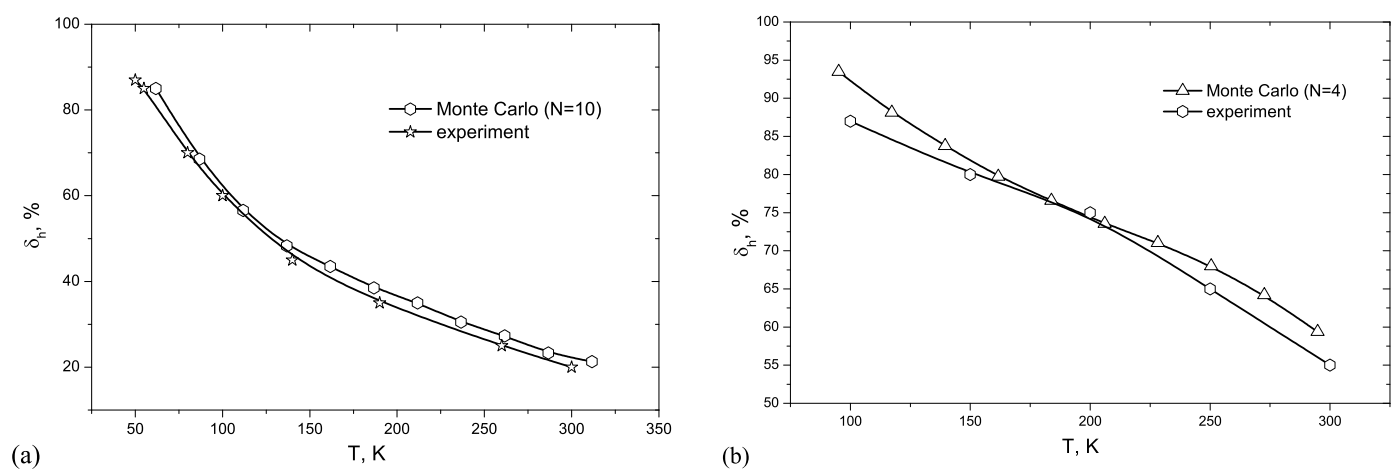

Fig. 5. Comparison of the calculated and experimentally measured in [3] temperature dependence of the magnetoresistance with the use of the ab initio determined values of the exchange integral for $\mathrm{Fe} / \mathrm{Cr}(100) / \mathrm{Fe}(\mathrm{a})$ and $\mathrm{Co} / \mathrm{Cu}(100) / \mathrm{Co}(\mathrm{b})$ structures

Also, we calculated the temperature dependence of the magnetoresistance for magnetic structures $\mathrm{Fe} / \mathrm{Cr}(100) / \mathrm{Fe}$ with $N=10$ and $\mathrm{Co} / \mathrm{Cu}(100) / \mathrm{Co}$ with $N=4$ given in Fig. 5 a,b with the use of the ab initio determined values of the exchange integral for ultrathin iron film $J_{1}=1.73 \cdot 10^{-14}, J_{2}=0.73 \cdot 10^{-14} \mathrm{erg}$ and cobalt film $J_{1}=2.52 \cdot 10^{-14} \mathrm{erg}, J_{2}=1.68 \cdot 10^{-14} \mathrm{erg}$. Comparison of calculated dependencies of the magnetoresistance for these structures with experimentally measured data shows also very well agreement.

Investigations were supported by Russian Science Foundation, project 14-12-00562.

\section{References}

[1] M.N.Baibich, J.M.Broto, A.Fert et al., Phys. Rev. Lett., 61(1988), 2472-2476.

[2] V.V.Prudnikov, P.V.Prudnikov, D.E.Romanovskiy, JETP Lett., 102(2015), 668-673. 
[3] J.Bass, W.P.Pratt, J. Magn. Magn. Mater., 200(1999), 274-289.

[4] P.V.Prudnikov, V.V.Prudnikov, M.A.Medvedeva, JETP Lett, 100(2014), 446-450.

\title{
Монте-Карло-моделирование магнитных мультислойных структур с эффектами гигантского магнитосопротивления
}

Дмитрий Е. Романовский

Марина В. Мамонова

Владимир В. Прудников

Павел В. Прудников

Омский государственный университет Мира, 55а, Омск, 644077

Россия

\begin{abstract}
Методами Монте-Карло осуществлено моделирование магнитных свойств структур, состоящих из двух ферромагнитных пленок, разделенных немагнитной пленкой с использовнием анизотропной модели Гейзенберга. Вычислено значение обменного интеграла для системы $\mathrm{Fe}(100) / \mathrm{Cr}$ с использованием РАW РВЕ приближения. Осуществлен расчет коэффициента магнитосопротивления в случае ТПП-геометрии для разных значений толщин ферромагнитных пленок. Показано, что рассчитанная температурная зависимость коэффициента магнитосопротивления находится в хорошем соответствии с экспериментальными данными, полученными для мультислойных структур $\mathrm{Fe} / \mathrm{Cr}$ and $\mathrm{Co} / \mathrm{Cu}$.

Ключевые слова: эффект гигантского магнитосопротивления, мультислойные магнитные структуры, ультратонкие пленки, коэфбициент магнитосопротивления, анизотропная модель Гейзенберга, методы Монте-Карло.
\end{abstract}

